\title{
Ensino personalizado: o MOODLE como ferramenta na busca da Aprendizagem Significativa
}

\author{
Kelly Hannel, PPGIE, UFRGS, khannel@ gmail.com \\ José Valdeni de Lima, PPGIE, UFRGS, valdeni@inf.ufrgs.br \\ Luís Descalço, Universidade de Aveiro, luisd@ua.pt
}

\begin{abstract}
Resumo. A educação personalizada só é possível após a identificação das dificuldades dos alunos que os impedem de terem os subsunçores necessários para captarem e aprenderem os conteúdos que lhes são ministrados. Com o uso das Tecnologias de Informação e Comunicação (TICs) e dos Ambientes Virtuais de Aprendizagem (AVAs), nesse artigo o MOODLE, são identificados os pontos fortes e fracos de cada aluno, relacionados nesse caso à disciplina de Língua Portuguesa do $6^{\circ}$ ano do Ensino Fundamental. Após essa identificação, são indicados conteúdos personalizados visando sanar as possíveis dificuldades de cada indivíduo. Constatou-se que através da indicação de Objetos de Aprendizagem (OAs) que levem em consideração o ritmo e a base de conhecimento individual, pode-se obter melhores resultados para a aprendizagem. Isso ocorre pois o aluno consegue ancorar os novos conteúdos a partir dos seus próprios subsunçores atingindo-se assim a almejada Aprendizagem Significativa.
\end{abstract}

Palavras chave: ensino personalizado, MOODLE, aprendizagem significativa, subsunçores.

\section{Personalized Learning: MOODLE as a tool in the search for Meaningful Learning}

\begin{abstract}
To have a personalized education we must identify students' difficulties that prevent them from having the subsumers necessary to capture and learn the content that they are taught. With the use of Information and Communication Technologies (ICTs) and Virtual Learning Environments (VLES), in this case the MOODLE, is possible to identify the strengths and weaknesses of each student, related to the discipline. In this paper, we worked with the discipline of Portuguese Language of the 6th year of elementary school. After this identification, personalized content are indicated aiming to remedy the possible difficulties of each individual. We came to conclusion that through the Learning Object indication (LOs) that take into account the pace and individual knowledge base we can get better results for the learning. This occurs because the student can anchor the new content from their own subsumers reaching up the Meaningful Learning.
\end{abstract}

Key words: personalized learning, MOODLE, meaningful learning, subsumers.

\section{INTRODUÇÃO}

As TICs são importantes no apoio ao desenvolvimento social e cognitivo dos indivíduos. A crescente necessidade de novas habilidades e conhecimentos trazem mudanças na percepção individual de aprendizagem e no sistema educacional com um 
todo. A mudança tecnológica implica profundas alterações na compreensão dos processos de interação social e na construção da aprendizagem e do conhecimento (Barros, 2011).

A Educação a Distância (EaD) quando baseada nas TICs é facilitada e melhorada através de AVAs, como o MOODLE, Blackboard, Rooda e o outros. Nestes ambientes os professores costumam postar os conteúdos e OAs para a turma toda, e não de acordo com as necessidades e preferências de cada aluno. A personalização, a comunicação e a colaboração que são possibilitadas através dos avanços da web tornamse um espaço de fundamental importância para o desenvolvimento de qualquer indivíduo. A evolução dos ambientes que podem ser utilizados para o ensino também têm acompanhado as melhorias que são trazidas pela web. Entretanto, o que se vê na maioria dos ambientes são OAs oferecidos para a turma toda, sem personalização ou direcionamento do aluno.

A aprendizagem personalizada é aquela que se adapta às necessidades particulares de um determinado estudante. Quando um estudante recebe atenção individualizada de um professor, geralmente, os resultados são superiores. Isto porque este professor ajusta sua explicação de acordo com o aluno, preservando o tempo e as necessidades dele. A abordagem personalizada implica que o aluno tenha atendimento individualizado quando necessitar, mas que também participe de projetos e atividades em grupo quando isto for facilitar o aprendizado (HORN e STAKER, 2015).

A personalização não é um conceito novo, diversos autores já abordaram o assunto, por exemplo: Keller (1968) e o Sistema Personalizado de Instrução, utilizados nas décadas de 60 e 70. A personalização parte do princípio de que as pessoas aprendem de formas diferentes e em ritmos diferentes, com base nos seus conhecimentos prévios, suas habilidades, seus interesses e emoções próprias (PORVIR..., 2015, p. 1).

Segundo Piaget (1996) os processos educacionais devem propor uma oportunidade para a descoberta e não simplesmente transmissão do conhecimento. Desta forma o aluno deve se sentir estimulado a explorar o objeto de aprendizagem. Neste contexto, quanto mais personalizado e focado o objeto for mais provável será o interesse do aluno.

Aliado ao conceito de oportunidade de descoberta de conhecimento, tem-se a teoria da aprendizagem significativa de Ausubel (1968), a qual preconiza que o aprender significativamente é ampliar e reconfigurar ideias já existentes na estrutura mental e com isso ser capaz de relacionar e acessar novos conteúdos. Quanto maior o número de links feitos, mais consolidado estará o conhecimento. Chegando-se assim a teoria de que quanto mais se sabe, mais se aprende. Ausubel (1968) considera a existência de conhecimentos prévios nos quais o estudante possa ancorar as novas ideias (quantidade e qualidade de subsunçores) como o fator de maior relevância para $\mathrm{o}$ aprendizado significativo. E, é nesse contexto que se enquadra esse trabalho. Visto que, o principal objetivo na personalização é identificar os subsunçores ou a ausência deles, para então indicar OAs capazes de sanar as dificuldades que impedem o aluno de aprender novos conteúdos.

Em uma turma presencial, muitas vezes, o professor consegue reconhecer as preferências e identificar possíveis deficiências de conteúdos em seus alunos (por exemplo, conteúdos pré-requisitos que o aluno esqueceu, ou mesmo não teve). Mas, esta é uma tarefa muito difícil, devido ao número de alunos que o mesmo professor precisa atender (Seed, 2005). 
Nesse trabalho, foram identificados os subsunçores, dentro da disciplina de Língua Portuguesa do $6^{\circ}$ ano do Ensino Fundamental (EF), que o aluno precisa dominar para então disponibilizar OAs que lhe permitam sanar possíveis dificuldades de conteúdo. Essa indicação de OAs foi realizada utilizando os recursos do MOODLE. A questão de pesquisa que buscou-se responder foi: "Como tornar a indicação de OAs personalizada, utilizando os recursos do MOODLE, para facilitar o aprendizado do aluno?"

O artigo está organizado como segue: na seção 2 são apresentados os métodos utilizados, na seção 3 é dedicado a uma breve explanação sobre os conceitos básicos de ensino híbrido e personalização, objetos de aprendizagem e do AVA MOODLE. Já na seção 4 é apresentado o relato da experiência realizada e a discussão dos resultados obtidos. Por fim, na seção 5 são apresentadas as conclusões e os trabalhos futuros.

\section{MÉTODOS}

Esta pesquisa está enquadrada como Pesquisa Qualitativa. A pesquisa qualitativa baseia-se na observação cuidadosa dos ambientes onde o sistema está sendo usado ou onde será usado, do entendimento das várias perspectivas dos usuários ou potenciais usuários do sistema (WAINER, 2007). Justifica-se a pesquisa qualitativa neste artigo pois a extração de dados descritos por meio dos conhecimentos externados pelos discentes no envolvimento com as atividades propostas no ambiente virtual. A técnica utilizada é o método estudo de caso exploratório e descritivo.

A metodologia foi divida em etapas sendo que algumas delas já foram cumpridas e outras estão em andamento ou serão realizadas futuramente. As etapas iniciais compreendem a revisão bibliográfica sobre os assuntos relacionados ao trabalho e a analise de abordagens similares. Posteriormente foi realizado um estudo de caso para verificar a validade das hipóteses levantadas. E como etapas futuras estão a descrição formal do modelo e testes com grupos maiores de alunos.

\section{REFERENCIAL TEÓRICO}

No sentido de cobrir os conceitos que fazem parte deste trabalho, as subseções seguintes apresentam as teorias que embasaram a metodologia e abordagens adotadas.

\subsection{Ensino híbrido e personalização}

Diversos trabalhos abordam o ensino híbrido e a personalização do ensino como uma forma de amenizar a distância cognitiva que se tem entre os alunos e também diminuir as dificuldades cognitivas. Por exemplo, Porvir (2015); Moran (2015), Horn e Staker (2015), dentre outros.

Segundo Christensen, et al. (2013, p.7):

“O ensino híbrido é um programa de educação formal no qual um aluno aprende, pelo menos em parte, por meio do ensino online. $\mathrm{O}$ estudante tem algum controle sobre pelo menos um dos seguintes elementos: tempo, lugar, modo e/ou ritmo do estudo. A educação ocorre pelo menos em parte em uma espaço físico supervisionado."

A personalização pode gerar diferenciação da forma de aprender na medida em que proporciona ao aluno um grau de escolha sobre o que é aprendido, quando se aprende e como aprende. Entretanto, os alunos ainda terão metas para cumprir. A ideia é 
fornecer a oportunidade de aprender de um modo que atenda às suas necessidades específicas (MILIBAND, 2006).

O ensino online pode massificar a questão de um professor por aluno. À medida que usa recursos tecnológicos para prover um ensino que atenda às necessidades de ritmo, dificuldades e tempo de cada aluno. Basicamente, o ensino online permite que o aluno avance rapidamente se já tiver assimilado o conteúdo, pare e estude novamente ou mesmo receba reforço naquele conteúdo caso não tenha entendido (aprendizado por maestria). Neste sentido os professores passam a ser planejadores, organizadores e mentores do que os alunos precisam estudar para atingir os objetivos. Entretanto, o simples fato de utilizar o ensino online não traz a personalização. É necessário que haja uma mudança de atitude tanto do professor quanto do aluno (HORN e STAKER, 2015).

O papel do professor passa a ser ativo, como um "designer de caminhos", de atividades individuais e em grupo. Sendo que a tecnologia traz a possibilidade de mesclar todos os espaços e tempos. Não são dois mundos ou espaços, mas sim um espaço estendido compreendido pela sala de aula física e a sala de aula online. Por isso a educação formal passa a ser mais "blended", misturada, híbrida. O professor precisa seguir comunicando-se presencialmente com os alunos, mas também precisa fazê-lo digitalmente. $\mathrm{O}$ aluno passa a ser produtor de informação, coautor com seus colegas e professores, reelaborando materiais, contando histórias, debatendo ideias em fóruns ou blogs, participando de webconferências (MORAN, 2015).

\subsection{Objetos de Aprendizagem}

Segundo Meyer (2008), OA é qualquer recurso digital utilizado nos processos de ensino e aprendizagem suportado por TICs. Nesta perspectiva, Papert (1994) define os objetos de aprendizagem como suportes concretos ou "objetos para pensar com" que auxiliam na construção do conhecimento pelo aluno. Através destas abordagens, o aluno desenvolve sua autonomia estimulada pela curiosidade que o impulsiona à pesquisa e ao desenvolvimento de suas habilidades.

\subsection{MOODLE}

Os AVAs tradicionais como MOODLE, Blackboard, Teleduc, Eduquito, Rooda são amplamente utilizados na EaD. Entretanto, normalmente, eles servem para atender todos os alunos da turma da mesma forma. Ou seja, o conteúdo e objetos de aprendizagem são os mesmos para todos os alunos. No geral, não são levadas em consideração informações do perfil ou necessidades específicas do aluno.

Para esse trabalho foi adotado o AVA MOODLE. Por ser um ambiente de código livre e ser um sistema de gerenciamento de aprendizagem com características, como adaptabilidade, adaptação, personalizável e extensível. Além de ser um dos mais populares sistemas de gerenciamento de aprendizagem baseado na web (GIRAFFA, 2009). Os cursos no MOODLE podem ter recursos como fóruns, chats, pesquisas e uma série de questionários. Pode ser utilizado em cursos de modalidade EaD, semipresenciais ou como ferramenta de comunicação, gerenciamento e organização de conteúdo em cursos presenciais. Além disso, possui diversos recursos e permite comunicação síncrona e assíncrona entre os participantes (SANTOS, 2011).

A partir da versão 2.0, o MOODLE permite aos professores mais flexibilidade na definição de caminhos de aprendizagem, com atividades condicionais. Essas atividades podem ser disponibilizadas aos alunos de acordo com determinadas condições, como: o grau obtido em um ou mais testes, a finalização de determinada 
atividade, ou uma combinação de condições. Normalmente, estas atividades são utilizadas para sugerir percursos mais adequados aos alunos (VEGALE, 2015). Um exemplo é a ferramenta lição, utilizada nesse trabalho e apresentada na seção 4.

\section{RELATO DE EXPERIÊNCIA}

Em uma escola pública de EF e Ensino Médio (EM) 45 alunos que cursavam o $6^{\circ}$ ano na disciplina de Língua Portuguesa realizaram um pré-teste e foram divididos em três grupos. Foi escolhida a disciplina de Língua Portuguesa, pois a direção da escola está buscando formas de melhorar a interpretação de textos,que é fundamental para o entendimento das questões de quaisquer outras disciplinas.

Foram realizados 8 encontros presenciais, de outubro à dezembro de 2015 e as atividades foram realizadas no MOODLE com supervisão e orientações da professora da disciplina. Nesse período foram implementados conceitos de ensino híbrido. Os alunos tiveram atividades que os desenvolviam como grupo, aplicando técnicas de aprendizado por maestria, organização por projetos, alunos executando rotação em sala de aula, etc. Por exemplo, foi realizada uma atividade de redação colaborativa. Também fizeram atividades em sala onde, enquanto um grupo trabalhava no computador preparando uma apresentação, outro trabalhava lendo um livro e outro fazia colagens e trabalhos manuais. Todos com a professora atendendo às necessidades individuais $\mathrm{e}$ circulando entre os grupos.

Para o pré-teste, que permitiu identificar os subsunçores iniciais dos estudantes, foi criado um curso no MOODLE e desenvolvida uma atividade questionário. Esta atividade consistiu de 20 questões de múltipla escolha, de respostas simples e de uma redação. Os resultados foram tabulados, com o auxílio de uma pedagoga, de acordo com os subsunçores definidos pela equipe pedagógica da escola e refletiam os conceitos estudados na série/ano em questão.

A divisão dos grupos ocorreu de acordo com os subsunçores apresentados na Tabela 1. Dos 45 alunos que fizeram o pré-teste, 12 tinham características do grupo 1, 18 se encaixavam no grupo 2 e 15 no grupo 3 . O grupo 1 era o mais básico, o grupo 2 o intermediário e o grupo 3 o avançado.

Tabela 1- Subsunçores de identificação dos grupos.

\begin{tabular}{|c|c|}
\hline GRUPOS & SUBSUNÇORES \\
\hline \multirow{3}{*}{$\begin{array}{l}\text { GRUPO } 1 \\
12 \text { alunos }\end{array}$} & Localizar informações explícitas em um texto. \\
\hline & Inferir o sentido de uma palavra a partir do contexto em que foi empregada. \\
\hline & Inferir o sentido de uma expressão a partir do contexto em que foi empregada. \\
\hline \multirow{4}{*}{$\begin{array}{l}\text { GRUPO } 2 \\
18 \text { alunos }\end{array}$} & Inferir uma informação implícita em um texto. \\
\hline & Identificar os elementos de um texto (narrador /foco narrativo). \\
\hline & Interpretar texto com auxílio de material gráfico diverso (propagandas, quadrinho, foto, etc.). \\
\hline & Identificar a finalidade de textos de diferentes gêneros. \\
\hline \multirow{3}{*}{$\begin{array}{c}\text { GRUPO } 3 \\
15 \text { alunos }\end{array}$} & $\begin{array}{l}\text { Estabelecer relações entre partes de um texto, identificando repetições ou substituições que } \\
\text { contribuem para a continuidade de um texto. }\end{array}$ \\
\hline & Estabelecer relação causa/consequência entre partes e elementos do texto. \\
\hline & $\begin{array}{l}\text { Reconhecer diferentes formas de tratar uma informação na comparação de textos que tratam } \\
\text { do mesmo tema, em função das condições em que ele foi produzido e daquelas em que será } \\
\text { recebido. }\end{array}$ \\
\hline
\end{tabular}

Fonte: Autores. 
Vislumbrando o uso da técnica estatística análise de variância com medidas repetidas de grupos foram subdivididos cada um dos grupos em controle e focal. Essa subdivisão foi realizada de forma aleatória e manteve-se o mesmo número de alunos no grupo participante do projeto e no grupo de controle. Ou seja, dos 12 alunos do grupo 1, 6 fizeram parte do focal e 6 do grupo de controle. O grupo 2 teve 9 alunos no grupo focal e 9 no grupo de controle e o grupo 3 teve 8 alunos no grupo focal e 7 no grupo de controle. Importante salientar que, tanto grupo de controle quanto o grupo focal tiveram aulas regulares com a professora e acessaram o MOODLE, a diferença é que os alunos do grupo focal recebiam atividades personalizadas e o grupo de controle não.

Após o pré-teste e a separação dos grupos, os alunos foram informados que receberiam atividades personalizadas e que haviam sido separados em grupos. Entretanto, não foram informados que estes grupos representavam níveis de aprendizagem, evitando assim que fossem criados "rótulos" e/ou os alunos pudessem ser estigmatizados pelos próprios colegas.

A versão do MOODLE utilizada na escola é a 2.8.1. Nesta versão os alunos poderiam ter sido agrupados e as atividades liberadas separadamente para cada grupo, ou como foi realizado, através da criação de três cursos, que foram chamado de Grupo 1, Grupo 2 e Grupo 3. Em cada curso foram cadastrados os alunos que receberiam as atividades personalizadas.

As aulas foram divididas em blocos, cada um com duração de uma ou duas semanas, dependendo da dificuldade do conteúdo e do ritmo de cada aluno. Eles não foram cobrados por tempo, e sim por executar a tarefa. Seguindo assim, a personalização do ritmo do aluno.

Foram selecionados OAs de diversos repositórios e a recomendação foi feita pelo próprio professor utilizando o MOODLE. De acordo com os resultados do pré-teste a professora recomendava os OAs aos alunos que faziam parte do grupo focal. Por exemplo, alunos que apresentaram dificuldade em acentuação gráfica recebiam acesso à atividades sobre este tema.

Para cada critério descrito na Tabela 1, foram selecionados objetos que o professor considerou que auxiliaria a sanar a dificuldade. E, foram desenvolvidos exercícios que privilegiassem a interpretação de textos. Contudo, não foram passados textos de forma convencional, e sim foram organizados textos e questões com diferentes níveis de dificuldade utilizando, principalmente a ferramenta lição do MOODLE.

A estrutura da ferramenta lição, desde que não configurada apenas como páginas web para navegação através de links, pode proporcionar o aprendizado por desempenho (ou maestria), isto é, de acordo com o que o aluno já sabe sobre determinado conteúdo. $\mathrm{O}$ estudante segue na sequência de perguntas de acordo com os seus acertos ou erros. Quando acerta a questão, o aluno segue por determinado conjunto de questões, mas se o aluno errar, segue por outro caminho; podendo inclusive voltar para a questão original. Outro ponto importante, na configuração da lição é a possibilidade de escolher uma dependência a uma lição anterior. Assim, o aluno só consegue avançar no curso se participar das lições anteriores. A atividade de "Leitura e interpretação" foi realizada utilizando a ferramenta lição e é mostrada na Figura 1. Nessa lição, os alunos recebiam uma atividade mais simples e iam passando para atividades mais complexas dependendo da sua evolução. Caso o aluno errasse determinada questão, ele recebia orientações sobre o assunto e retornava para as questões iniciais. 


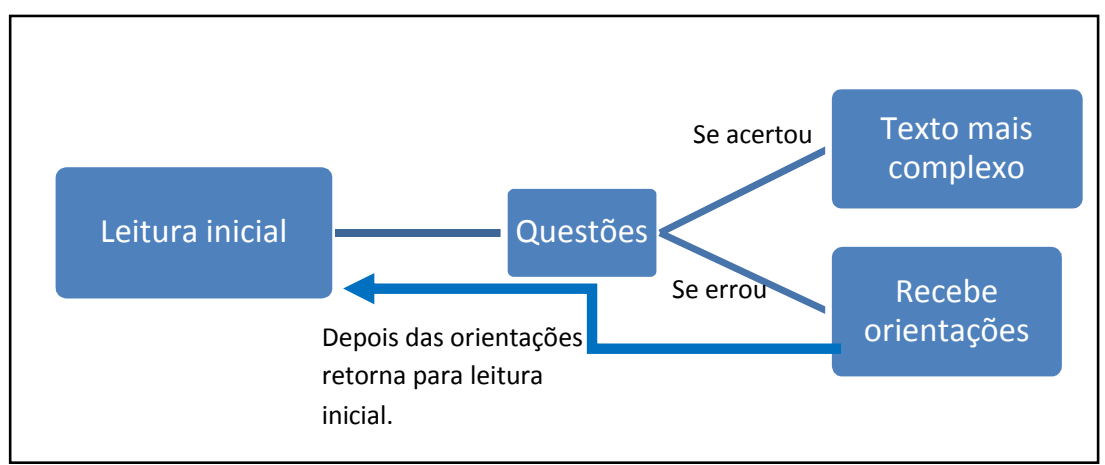

Figura 1- Atividade de leitura e interpretação utilizando a ferramenta lição.

Fonte: Autores.

Após um período de oito semanas os alunos foram submetidos a um pós-teste. $\mathrm{O}$ pós-teste também foi composto de 20 questões de múltipla escolha e uma redação. Os níveis de dificuldades das questões aplicadas nas avaliações foram os mesmos tanto no pré-teste quanto no pós-teste.A comparação entre o pré e pós-teste representa a evolução dos alunos. Na Figura 2 é apresentado o gráfico com esta evolução. A partir deste gráfico é possível observar que tanto os alunos que receberam indicação de OAs personalizados quanto o grupo de controle evoluíram. Entretanto, os que tiveram um tratamento personalizado tiveram uma magnitude de evolução maior. E, quando observados os subsunçores que geraram as recomendações, é possível notar que os alunos que receberam OAs personalizados não mais apresentaram as mesmas dificuldades. Mostrando com isso, que a recomendação de OAs de acordo com as necessidades levou a um aprendizado mais efetivo.

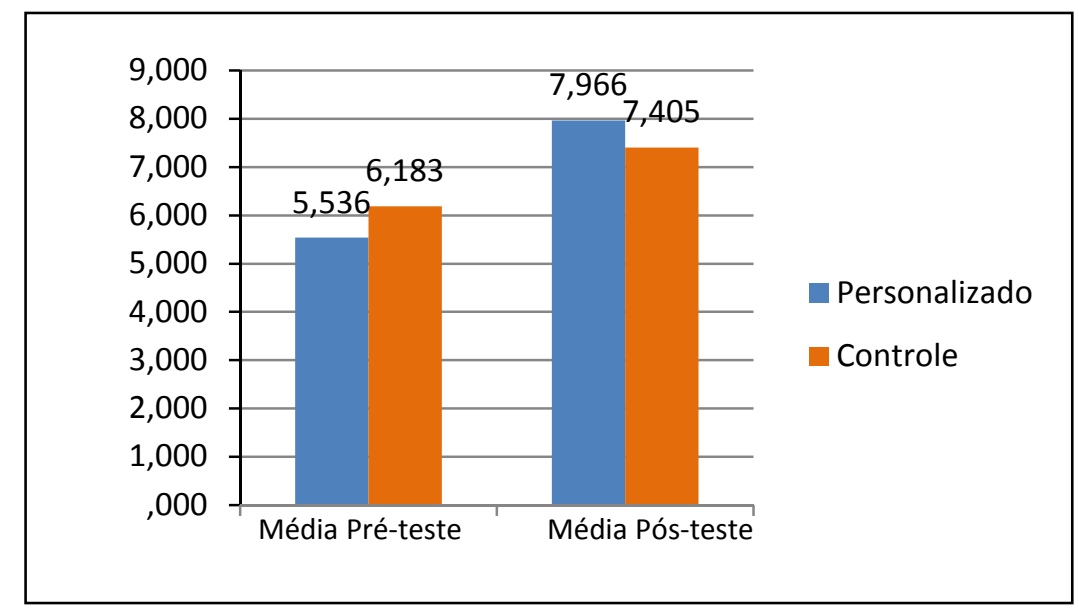

Figura 2- Evolução dos alunos que receberam OAs de acordo com as suas dificuldades $\mathrm{X}$ grupo de controle.

Fonte: Autores.

A Tabela 2 resume, para cada aluno, o grupo ao qual pertencia no pré-teste, o grupo no pós-teste e também se fazia parte grupo focal ou de controle. 
Tabela 2- Evolução de grupo por aluno.

\begin{tabular}{|c|c|c|c|}
\hline \multicolumn{4}{|c|}{ Comparação da mudança de grupo } \\
\hline Aluno & AD1 & AD2 & Grupo \\
\hline 1 & Grupo 1 & Grupo 2 & Projeto \\
\hline 2 & Grupo 1 & Grupo 2 & Projeto \\
\hline 3 & Grupo 1 & Grupo 2 & Projeto \\
\hline 4 & Grupo 2 & Transferido de escola & Controle \\
\hline 5 & Grupo 1 & Grupo 2 & Controle \\
\hline 6 & Grupo 2 & Grupo 2 & Controle \\
\hline 7 & Grupo 2 & Grupo 3 & Projeto \\
\hline 8 & Grupo 2 & Grupo 3 & Projeto \\
\hline 9 & Grupo 2 & Grupo 3 & Projeto \\
\hline 10 & Grupo 2 & Transferido de escola & Controle \\
\hline 11 & Grupo 2 & Grupo 2 & Controle \\
\hline 12 & Grupo 3 & Grupo 3 & Controle \\
\hline 13 & Grupo 1 & Grupo 2 & Projeto \\
\hline 14 & Grupo 2 & Grupo 3 & Projeto \\
\hline 15 & Grupo 2 & Grupo 3 & Projeto \\
\hline 16 & Grupo 1 & Grupo 1 & Controle \\
\hline 17 & Grupo 1 & Grupo 2 & Controle \\
\hline 18 & Grupo 2 & Grupo 2 & Projeto \\
\hline 19 & Grupo 2 & Grupo 3 & Projeto \\
\hline 20 & Grupo 1 & Grupo 2 & Projeto \\
\hline 21 & Grupo 2 & Grupo 2 & Projeto \\
\hline 22 & Grupo 3 & Grupo 3 & Projeto \\
\hline 23 & Grupo 3 & Grupo 3 & Projeto \\
\hline 24 & Grupo 3 & Grupo 3 & Projeto \\
\hline 25 & Grupo 3 & Grupo 3 & Projeto \\
\hline 26 & Grupo 2 & Grupo 3 & Projeto \\
\hline 27 & Grupo 2 & Grupo 2 & Controle \\
\hline 28 & Grupo 1 & Grupo 3 & Projeto \\
\hline 29 & Grupo 3 & Grupo 3 & Projeto \\
\hline 30 & Grupo 2 & Grupo 2 & Controle \\
\hline 31 & Grupo 2 & Grupo 2 & Controle \\
\hline 32 & Grupo 2 & Grupo 2 & Controle \\
\hline 33 & Grupo 2 & Grupo 2 & Controle \\
\hline 34 & Grupo 2 & Grupo 2 & Controle \\
\hline 35 & Grupo 2 & Grupo 2 & Controle \\
\hline 36 & Grupo 3 & Grupo 2 & Controle \\
\hline 37 & Grupo 2 & Grupo 3 & Controle \\
\hline 38 & Grupo 2 & Grupo 2 & Controle \\
\hline 39 & Grupo 2 & Grupo 2 & Controle \\
\hline 40 & Grupo 2 & Grupo 1 & Controle \\
\hline
\end{tabular}




\begin{tabular}{|l|l|l|l|}
41 & Grupo 3 & Grupo 3 & Projeto \\
\hline 42 & Grupo 3 & Grupo 3 & Projeto \\
\hline 43 & Grupo 3 & Grupo 3 & Projeto \\
\hline 44 & Grupo 3 & Grupo 3 & Controle \\
\hline 45 & Grupo 3 & Grupo 3 & Controle \\
\hline
\end{tabular}

Fonte: Autores.

Os resultados obtidos com a comparação dos subsunçores utilizados demonstraram que:

- Dos alunos que participaram do projeto: 21 alunos evoluíram; 2 mantiveram as mesmas dificuldades e nenhum piorou de rendimento. Ou seja, 91\% dos alunos tiveram uma diminuição considerável nas dificuldades apresentadas;

- Dos alunos do grupo de controle: 6 alunos evoluíram;12 alunos permaneceram apresentando as mesmas dificuldades; 2 alunos tiveram uma piora nas dificuldades apresentadas. Ou seja, 30\% dos alunos apresentaram evolução.

Uma importante constatação para identificar se o aluno teve uma real evolução de aprendizado, é o fato dele deixar de errar questões que envolvem os subsunçores do seu grupo e assim poder trocar de grupo, para um que possua subsunçores mais avançados.

\section{CONCLUSÕES E TRABALHOS FUTUROS}

Esse artigo apresentou um trabalho desenvolvido em uma escola pública de EF e EM. A ideia principal foi servir como base para comprovar que quando os alunos recebem OAs personalizados visando obter os subsunçores necessários para entender os conteúdos ministrados pelo professor o aprendizado é mais significativo. Nesse sentido, foi desenvolvido um experimento, onde os alunos passaram por um pré-teste, foram divididos em dois grupos, o focal que recebeu OAs de acordo com as suas dificuldades e outro que serviu de grupo de controle. Ainda, dentro do grupo focal, foram subdivididos em 3 grupos de acordo com os subsunçores identificados. Após um período de atividades, os alunos passaram por um pós-teste que trouxe excelentes resultados.

A evolução dos alunos, quando considerados os seus subsunçores presentes ou não, demonstram resultados animadores. Quando se trata de alunos que receberam OAs personalizados, $91 \%$ dos alunos evoluíram, em contrapartida, somente $30 \%$ evoluiu quando se trata dos alunos que fizeram parte do grupo de controle. Esses resultados fizeram que a direção da escola permitisse e incentivasse a continuação dos estudos.

As atividades subsequentes estão sendo a identificação de subsunçores necessários em outras disciplinas e aplicação das técnicas desenvolvidas e utilizadas neste artigo em um número maior de alunos. No segundo semestre de 2016, 180 alunos que cursam matemática no $1^{\circ}$ ano do ensino médio da mesma escola, estão recebendo OAs personalizados. Além disso, pretende-se formalizar o modelo para replicação em outras escolas. 


\section{REFERENCIAL BIBLIOGRÁFICO}

AUSUBEL, D. P. (1968). Educational psychology: a cognitive view. New York, Holt, Rinehart and Winston.

BARROS, D. M. V. et. al. (2011) Educação e tecnologias: reflexão, inovação e práticas. Lisboa: [s..n.]

CHRISTENSEN, C. M., HORN, M. B., STAKER, H. Ensino Híbrido: uma inovação disruptiva? Uma introdução à teoria dos híbridos. [S.1: s. n]. Disponível em: $<$ http://porvir.org/wp-content/uploads/2014/08/PT_Is-K-12-blended-learningdisruptive-Final.pdf $>$. Acesso em: 23 out 2016.

GIRAFFA, L. M. M. Uma odisséia no ciberespaço: O software educacional dos tutoriais aos mundos virtuais. Revista Brasileira de Informática na Educação. [S.1], v. 17, n. 01, p. 20, 2009.

HORN, M. B.; STAKER, H. Blended: Usando a Inovação Disruptiva para Aprimorar a Educação. Porto Alegre: Penso, 2015.

KELLER, F. S. (1968). "Good-Bye teacher...". Journal of applied behavior analysis, 1968 - 1.p 79-89. Wiley Online Library.

MEYER, M. Modularization and Multi-Granularity Reuse of Learning Resources. 2008. pp 19-23. Disponível em: <http://tuprints.ulb.tu-darmstadt.de/1156 >. Acesso em 16 out 2013.

MILIBAND, D. Choice and Voice in Personalised Learning. In: Personalising Education. OECD, 2006, p. 24

MORAN, J. Educação Híbrida Um conceito chave para a educação, hoje. In: Bacich, L.; Tanzi Neto, A.; Trevisani, F. M. (Orgs). Ensino híbrido: personalização e tecnologia na educação. - Porto Alegre: Penso, 2015.

PAPERT, S. (1994). A máquina das crianças: repensando a escola na era da informática. Porto Alegre: Artes Médicas.

PIAGET, J. Biologia e conhecimento. 2a . edição. Petrópolis: Vozes, 1996.

PORVIR: o futuro se aprende. Personalização. 2015. Disponível em: < http://www.porvir.org/especiais/personalizacao/>. Acesso em: 1 mar 2016.

SANTOS, N. ET. AL. Monitoramento navegacional do aluno para descoberta de padrões de preferências de aprendizagem no MOODLE. Porto Alegre: Cadernos de Informática, v.6, n. 01, 2011.

SEED., "Integração das Tecnologias na Educação/ Secretaria de Educação a Distância". Brasília: Ministério da Educação, Seed, 2005. Disponível em: $<$ http://tvescola.mec.gov.br/images/stories/publicacoes/salto_para_o_futuro/livro_sal to_tecnologias.pdf>. Acesso em: 14 out 2013.

VAGALE, V. 2015.Personalization Opportunities in the MOODLE System. Disponível em: $<$ http://www.academia.edu/3275982/PERSONALIZATION_OPPORTUNITIES _IN_THE_MOODLE_SYSTEM>. Acesso em: 22 Fev. 2016.

WAINER, J.. Métodos de pesquisa quantitativa e qualitativa para a ciência computação. In: Tomaz Kowaltowski; Karin Breitman. (Org.). Atualização em informática. Sociedade Brasileira de Computação e Editora PUC Rio, 2007, v. 1, p. 221-262. 